\title{
Quantum chemical studies on some potentially tautomeric thiazolidinone derivatives and their thio and azo analogs
}

\author{
Nesrin Tokay ${ }^{a}$ and Cemil Öğretir ${ }^{b} *$ \\ ${ }^{a}$ Hacettepe University, Faculty of Science, Chemistry Department, 06532 Beytepe-Ankara, \\ Turkey \\ ${ }^{b}$ Faculty of Arts and Sciences, Chemistry Department, Eskişehir Osmangazi University, 26040 \\ Eskişehir, Turkey \\ E-mail: cogretir@,ogu.edu.tr
}

\begin{abstract}
The gas phase semi-empirically calculated relative stability and tautomeric equilibrium values revealed that $2 \mathrm{C}, 4 \mathrm{C}$, and $5 \mathrm{C}$ hydroxy substituted thiazolidinone derivatives prefer oxo forms. The $2 \mathrm{C}, 4 \mathrm{C}$, and $5 \mathrm{C}$ mercapto substituted derivatives also found to prefer the thione forms. On the contrary $2 \mathrm{C}, 4 \mathrm{C}$, and $5 \mathrm{C}$ amino substituted derivatives were found to prefer amino forms.
\end{abstract}

Keywords: Quantum calculations, tautomerism, hydroxythiazolidine, mercaptothiazolidine, aminothiazolidine

\section{Introduction}

It has been well known that thiazole derivatives play very important roles in biochemical reactions such as oxidative decarboxylation and formation of $\alpha$-hydroxyalkyl ketons (i.e. structural change) called acyloins. ${ }^{1}$ It seems that there exists no systematic theoretical work on the tautomerism of potentially tautomeric thiazole derivatives. We believe that this gap should be filled up to a certain extent by studying the tautomeric equilibria of some potentially tautomeric thiazole derivatives therefore following our work on tautomerism, isomerism and deprotonation of some 5(6)-substituted benzimidazole-2-thione derivatives ${ }^{2}$ we now reporting on some quantum chemical studies on potentially tautomeric thiazolidinone derivatives and their thio and azo analogs.

The knowledge of the acidity and tautomeric structure of ionizable molecules are important for understanding of many areas of chemistry both in the gas phase and solution. Therefore, it is necessary to calculate the acidity constants, $\mathrm{pK}_{\mathrm{a}}$ values, and tautomeric equilibrium constants, $\mathrm{pK}_{\mathrm{T}}$ values. They are of particular interest for elucidating reaction mechanisms, especially those having proton transfers, and for interpreting the binding of substrates or inhibitors to enzymes. 
However, experimental determinations of individual $\mathrm{pK}_{\mathrm{a}}$ values are difficult in complex systems and kinetic assignments of $\mathrm{pK}_{\mathrm{a}}$ 's are often complicated by uncertainties in interpreting the $\mathrm{pH}$ dependence of the measured parameters. It is therefore useful to have reliable and accurate means of calculating relative and/or absolute $\mathrm{pK}_{\mathrm{a}}$ values and to have an understanding is essential for interpreting the measured effective $\mathrm{pK}_{\mathrm{a}}$ values in complex poly ions. ${ }^{3}$

\section{Methods of calculation}

Geometry optimizations were carried out at semi empirical MNDO, ${ }^{4} \mathrm{AM} 1,{ }^{5}$ and $\mathrm{PM} 3{ }^{6}$ levels of theory at the restricted Hartree-Fock level (RHF). The calculations were performed using MOPAC 7.0 program package. ${ }^{7}$ The solvent effect was included in the geometry optimizations following the 'COnductor-like Screening MOdel' (COSMO) ${ }^{8}$ implemented in MOPAC 7.0.

The MOPAC 7.0 was used to obtain values of thermo chemical and physicochemical quantities. Initial geometries of all structures were obtained by a molecular mechanics program (Chem Office Pro for Windows) ${ }^{9}$ followed by full optimization of all geometric variables without any symmetry constraint using four semi-empirical methods in MOPAC 7.0 and CACHE programs. ${ }^{6,10}$

The acidity of a given base, B, for the protonation reaction (Eq.1) in which $\mathrm{n}=1$ for protonation of a neutral base, can be calculated using the Eq. 2 where $\Delta \mathrm{G}$ is the standard free energy $^{11} \mathrm{~B}$ and $\mathrm{BH}^{+}$are neutral and protonated base $\mathrm{B}$ respectively. The computed thermodynamic data of various species were used in predicting the acidity constants, $\mathrm{pK}_{\mathrm{a}}$ values, using Eq.3 in which the $\delta \Delta \mathrm{G}_{\mathrm{BH}}{ }^{+}$is the standard free energy change for protonation reaction (Eq.1).

$$
\begin{gathered}
\mathrm{B}^{(\mathrm{n}-1)+}+\mathrm{H}_{3} \mathrm{O}^{+} \rightleftharpoons \mathrm{BH}^{\mathrm{n}+}+\mathrm{H}_{2} \mathrm{O} \\
\left.\delta \Delta \mathrm{G}_{\mathrm{f}(\mathrm{BH}}^{+}\right)=\left[\Delta \mathrm{G}_{\mathrm{f}(\mathrm{B})}+\Delta \mathrm{G}_{\mathrm{f}\left(\mathrm{H}^{+}\right)}=\left[\Delta \mathrm{G}_{\mathrm{f}\left(\mathrm{BH}^{+}\right)}^{+}+\Delta \mathrm{G}_{\mathrm{f}(\mathrm{H} 2 \mathrm{O})}\right]\right. \\
\left.\mathrm{pK}_{\mathrm{a}}=\delta \Delta \mathrm{G}_{\mathrm{f}(\mathrm{BH}}^{+}\right) / 2.303 \mathrm{RT}
\end{gathered}
$$

Carlton's equation may let us to calculate the tautomeric equilibrium constants by using the acidity constants, $\mathrm{pK}_{\mathrm{a}}$ values, of the model compounds in the following manner. ${ }^{13}$

$$
\begin{gathered}
\text { Keto } \stackrel{\mathrm{K}_{\mathrm{T}}}{\rightleftharpoons} \text { Enol } \\
\text { Thione } \stackrel{\stackrel{\mathrm{K}_{\mathrm{T}}}{\rightleftharpoons}}{\rightleftharpoons} \text { Thiole } \\
\text { Imino } \stackrel{\mathrm{K}_{\mathrm{T}}}{\rightleftharpoons} \text { Amino } \\
\left.\left.\mathrm{pK}_{\mathrm{T}}=\mathrm{pK}_{\mathrm{a}} \text { (model for product }\right)-\mathrm{pK}_{\mathrm{a}} \text { (model for reactant }\right)
\end{gathered}
$$




\section{Results and Discussion}

The nomenclature of the studied molecules was given in Table 1. The gas phase and aqueous phase MNDO, AM1, PM3, and PM5 computed data and calculated physical parameters were depicted in Tables 2-5.

\section{Stability and tautomerisation}

When a potentially tautomeric hydroxy group is placed at $2 \mathrm{C}, 4 \mathrm{C}$ and $5 \mathrm{C}$ (molecules $\mathbf{1}$, 2, and $\mathbf{3}$ ) of thiazolidin molecules the gas phase stability studies with MNDO, AM1, PM3, and PM5 methods has indicated that the thione forms a are favored over the enol forms $\mathbf{b}$ in ring-chain tautomerism (Scheme 1) as reported in the literature. ${ }^{15,16}$

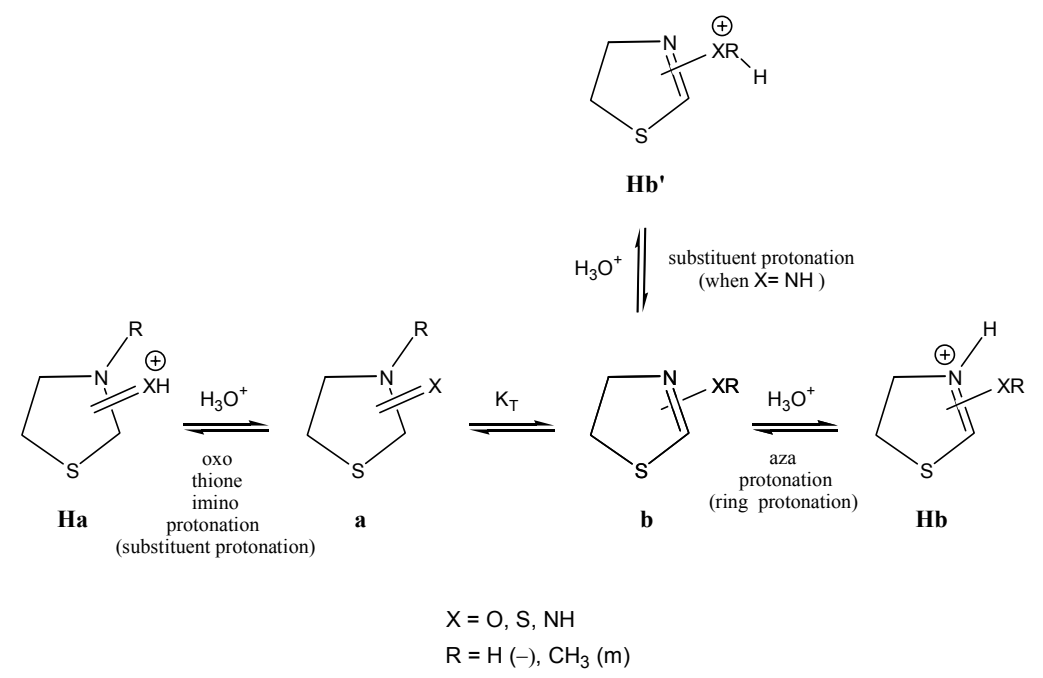

Scheme 1. Possible ring-chain tautomerisation and protonation patterns for studied thiazoldinones and their thio and aza analogs.

When a potentially tautomeric mercapto group is placed at $2 \mathrm{C}, 4 \mathrm{C}$, and $5 \mathrm{C}$ (molecules $\mathbf{4}, \mathbf{5}$, and 6) of thiazolidine molecules the gas phase stability studies with all four methods indicate that the main tautomers exist in the thione forms a rather than thiol forms $\mathbf{b}$. Model molecules were found to behave similar to main molecules and they prefer to exist mainly in thione forms a rather than thiol forms $\mathbf{b}$ (i. e. $\mathrm{RS}>0$ ) also as reported earlier for aromatic analogs. ${ }^{16,17}$

When a potentially tautomeric amino group is placed at $2 \mathrm{C}, 4 \mathrm{C}$, and $5 \mathrm{C}$ (molecules 7, 8 and 9 ) of thiazolidine molecule the gas phase stability values of MNDO, AM1, PM3, and PM5 methods indicate amino forms $\mathbf{b}$ are favored over the imino forms $\mathbf{a}$ (i. e. $\mathrm{RS}<0$ ) ) as indicated in the literature. ${ }^{18}$ 
Table 1. Formulae and nomenclature of the studied molecules

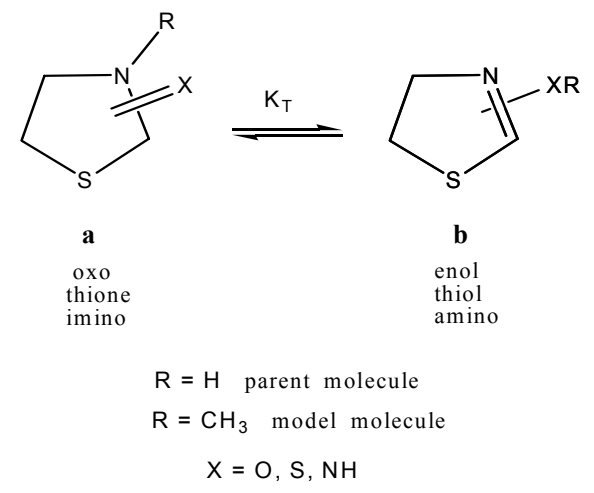

\begin{tabular}{|c|c|c|c|}
\hline Molecule & $\begin{array}{c}\text { Name } \\
\text { for parent compounds }\end{array}$ & Molecule & $\begin{array}{c}\text { Name } \\
\text { for model molecules }\end{array}$ \\
\hline \multicolumn{2}{|r|}{$\mathbf{R}: \mathbf{H}$} & \multicolumn{2}{|r|}{$\mathrm{R}: \mathrm{CH}_{3}$} \\
\hline \multicolumn{4}{|c|}{$\mathbf{X}: \mathbf{O}$} \\
\hline 1a & Thiazolidin-2-one & m1a & $N$-Methyl thiazolidin-2-one \\
\hline $1 b$ & 2-Hydroxy thiazolidine & m1b & 2-Methoxy thiazolidine \\
\hline $\mathbf{2 a}$ & Thiazolidin-4-one & $\mathbf{m} 2 \mathbf{a}$ & $N$-Methyl thiazolidin-4-one \\
\hline $\mathbf{2 b}$ & 4-Hydroxy thiazolidine & $\mathbf{m} 2 \mathbf{b}$ & 4-Methoxy thiazolidine \\
\hline $3 \mathbf{a}$ & Thiazolidin-5-one & m3a & $N$-Methyl thiazolidin-5-one \\
\hline $\mathbf{3 b}$ & 5-Hydroxy thiazolidine & $\mathbf{m 3 b}$ & 5-Methoxy thiazolidine \\
\hline \multicolumn{4}{|c|}{$X: S$} \\
\hline $4 \mathbf{a}$ & Thiazolidin-2-thione & $\mathbf{m 4 a}$ & $N$-Methyl thiazolidin-2-thione \\
\hline $4 b$ & 2-Mercapto thiazolidine & $\mathbf{m} 4 \mathbf{b}$ & 2-Mercapto methyl thiazolidine \\
\hline $5 \mathbf{a}$ & Thiazolidin-4-thione & $\mathbf{m 5 a}$ & $N$-Methyl thiazolidin-4-thione \\
\hline $5 \mathbf{b}$ & 4-Mercapto thiazolidine & m5b & 4-Mercapto methyl thiazolidine \\
\hline $6 \mathbf{a}$ & Thiazolidin-5-thione & m6a & $N$-Methyl thiazolidin-5-thione \\
\hline $6 \mathbf{b}$ & 5-Mercapto thiazolidine & m6b & 5-Mercapto methyl thiazolidine \\
\hline \multicolumn{4}{|c|}{$\mathbf{X}: \mathbf{N H}$} \\
\hline $7 \mathbf{a}$ & 2-Iminothiyolidine & $\mathbf{m} 7 \mathbf{a}$ & 2-Methylaminothiyolidine \\
\hline $7 \mathbf{b}$ & 2-Aminothiolidine & $\mathbf{m} 7 \mathbf{b}$ & 2-Dimethylaminothiyolidine \\
\hline \multicolumn{4}{|c|}{$\mathbf{m} 7 \mathbf{b}^{\prime}$} \\
\hline $\mathbf{8 a}$ & 4-Iminothiyolidine & m8a & 4-Methylaminothiyolidine \\
\hline $\mathbf{8 b}$ & 4-Aminothiyolidine & $\mathbf{m 8 b}$ & 4-Dimethylaminothiyolidine \\
\hline \multicolumn{4}{|c|}{ m8b' } \\
\hline $9 \mathbf{a}$ & 5-Iminothiyolidine & $\mathbf{m 9 a}$ & 5-Methylaminothiyolidine \\
\hline $9 \mathbf{b}$ & 5-Aminothiyolidine & m9b & 5-Dimethylaminothiyolidine \\
\hline \multicolumn{4}{|c|}{ m9b' } \\
\hline
\end{tabular}




\section{Acidity constants}

To be able to reach a conclusion about the closeness of theoretically calculated acidity constants which were depicted in Table 5 to the reality we have tried to compare those results with the experimentally obtained acidity constants for analogous molecules.

For 4-methyl-2-oxothiazole and its $\mathrm{N}$-methyl model for example the oxo protonation $\mathrm{pK}_{\mathrm{a}}$ values were reported as -1.80 and -1.70 respectively. ${ }^{12}$ Therefore, for molecule 1a under study the AM1 calculated $\mathrm{pK}_{\mathrm{a}}$ value of -2.29 and for its $\mathrm{N}$-methyl model $\mathbf{m 1 a} \mathrm{pK}_{\mathrm{a}}$ value of -3.46 suggest a similar protonation pattern and its oxo protonation as in the case of aromatic analogous. This behavior of molecule $\mathbf{1}$ is also indicative of the stability of oxo form, 1a, with comparing the enol form, $\mathbf{1 b}$.

A similar conclusion can be withdrawn for 4-oxo and 5-oxo derivatives (i.e. molecules 2 and 3). The AM1 calculated $\mathrm{pK}_{\mathrm{a}}$ values of -2.86 and -3.42 for molecule 2 and its $\mathrm{N}$-methyl model respectively, in which proton migration was eliminated, $\mathbf{m} 2$ suggests similar protonation pattern and that is oxo protonation. In same way we can say study the AM1 calculated $\mathrm{pK}_{\mathrm{a}}$ values of 6.27 and -7.32 for molecule $\mathbf{3}$ and its $\mathrm{N}$-methyl model respectively, $\mathbf{m} 3$, suggest that the protonation occurs at the oxo group and oxo form is stable.

For thiazolidin-2-thione, $\mathbf{4 a}$, and for its $\mathrm{N}$-methyl model, m4a, the AM1 calculated $\mathrm{pK}_{\mathrm{a}}$ values were found as -3.80 and -2.05 respectively. These values are very close to their oxo analogs and suggest thione protonation. These values also suggest the stability of thione forms. For 5-mercaptothiazolidine, 5b, and its mercapto methyl model, $\mathbf{m 5} \mathbf{b}$, the PM5 calculated $\mathrm{pK}_{\mathrm{a}}$ values seem to be reliable (i. e. -0.65 and -1.64 respectively).

For 4-mercapto thiazolidine, $\mathbf{6 b}$, and its mercapto methyl model, m6b, have the MNDO calculated $\mathrm{pK}_{\mathrm{a}}$ values of -3.88 and -4.14 which are close to oxo analogs. These values are indicative of ring protonation (i.e. nitrogen atom protonation at N3 of the thiazlodin-4-thione ring). Indication the stability of mercapto form $\mathbf{6 b}$.

For 2-aminothiazolidine, $\mathbf{7 b}$, and for its $\mathrm{N}$-methyl derivate, $\mathbf{m} \mathbf{7 b}$, the AM1 calculated $\mathrm{pK}_{\mathrm{a}}$ values were found as 8.45 and 2.29 seem to be realistic and closer to experimentally obtained values of aromatic analogs. ${ }^{11}$ The lowering of basicity, of course, can be attributed to tertiary amine form of $\mathbf{m} \mathbf{7 b}$. So the protonation takes place at the amino group and the amino form is more stable for $\mathbf{7 b}$. Similarly for 4-aminothiazolidine, $\mathbf{8 b}$, and for its N-methyl model, $\mathbf{m} \mathbf{8 b}$, we can say that the protonation takes place on the amino group and the AM1 calculated $\mathrm{pK}_{\mathrm{a}}$ values of 16.06 and 12.56 suggest the basicity lowering, and amino form is more stable. For 5aminothiazolidine, $\mathbf{8 b}$, and for its N-methyl model, $\mathbf{m 8 b}$ ', and the PM5 calculated $\mathrm{pK}_{\mathrm{a}}$ values of 8.07 and 6.66 seem to be realistic suggesting the stability of amino tautomer, $\mathbf{8 b}$, and amino protonation lowering of basicity about $2 \mathrm{pK}_{\mathrm{a}}$ unit is expected.

\section{Tautomerism}

As indicated earlier, acidity constant measurement is very important. The aqueous phase calculated tautomeric equilibrium constants for the main tautomers with all four methods (i.e. MNDO, AM1, PM3, and PM5) indicate the stability of keto and thione forms a over the enol and 
thiol forms $\mathbf{b}$ when the hydroxy and mercapto groups are placed at $2 \mathrm{C}, 4 \mathrm{C}$, and $5 \mathrm{C}$ of the thiazolidine ring (i. e. $\mathrm{pK}_{\mathrm{T}}>0$ ). The model molecule studies for 2- or 4-mercapto derivatives however suggest reverse indicating the stability of thiol form $\mathbf{b}$ over the thione form $\mathbf{a}$ (i. e. $\mathrm{pK}_{\mathrm{T}}$ $<0)$ as it happened in stability studies.

In elucidation of reaction mechanism such as protonation. They are also being used in determination of tautomeric equilibrium. Usage of Charton's equation in these calculations are invaluable. In the present work we made use of this equation in interpreting the obtained results (Tables 3-4).

When a potentially tautomeric amino group is placed at $2 \mathrm{C}$ or $4 \mathrm{C}$ of thiazolidine molecule both main molecules and fixed models aqueous tautomeric constants, $\mathrm{K}_{\mathrm{T}}$ values of MNDO, AM1, and PM3 methods indicate that amino forms $\mathbf{b}$ are favored over the imino forms $\mathbf{a}$ (i. e. $\left.\mathrm{pK}_{\mathrm{T}}<0\right)$.

\section{Correlation search}

Due to the lack of experimentally determined acidity constants, $\mathrm{pK}_{\mathrm{a}}$ values, for the studied compounds we had to search some correlations to be sure that the predicted values are close to the real values. To achieve this goal, correlations among the calculated $\mathrm{pK}_{\mathrm{a}}$ values with different methods were compared. Correlation graphs of $\mathrm{pK}_{\mathrm{a}}$ vs. $\mathrm{pK}_{\mathrm{a}}, \mathrm{pK}_{\mathrm{a}}$ vs. nucleophilicity, $\mathrm{pK}_{\mathrm{T}} \mathrm{vs}_{\mathrm{p}} \mathrm{pK}_{\mathrm{T}}$ are depicted in Figures 1-3. the best correlation was obtained between the PM5 and PM3 calculated $\mathrm{pK}_{\mathrm{a}}$ values. However, the best correlation AM1 calculated nucleophilicity and PM3 calculated nucleophilicity was obtained. The best fit to tautomeric equlibrium constants, $\mathrm{pK}_{\mathrm{T}}\left(\mathrm{PM}\right.$ ) values comes from the $\mathrm{pK}_{\mathrm{T}}(\mathrm{AM} 1)$ values.

Table 2. Gas phase relative stabilities $\left(\mathrm{RS}^{\mathrm{a}}{ }^{\mathrm{b}}\right.$, $\left.\mathrm{kcal} / \mathrm{mol}\right)$ of the thiazolidine derivatives

\begin{tabular}{rrrrr}
\hline & \multicolumn{4}{c}{$\mathrm{RS}^{\mathrm{a}}\left(\mathrm{kcal} \mathrm{mol}^{-1}\right)$} \\
\cline { 2 - 5 } $\mathbf{1 a}=\mathbf{1 b}$ & $\mathrm{MNDO}$ & $\mathrm{AM} 1$ & $\mathrm{PM} 3$ & $\mathrm{PM5}$ \\
\cline { 2 - 5 } $\mathbf{2 a}=\mathbf{2 b}$ & -3.64 & -12.07 & -9.09 & -12.95 \\
$\mathbf{3 a}=\mathbf{3 b}$ & -9.13 & -9.22 & -10.74 & -15.61 \\
$\mathbf{4 a}=\mathbf{4 b}$ & 3.49 & -4.13 & -8.81 & -7.51 \\
$\mathbf{5 a}=\mathbf{5 b}$ & -1.54 & -0.94 & 7.88 & -8.63 \\
$\mathbf{6 a}=\mathbf{6 b}$ & 2.31 & -1.49 & 6.80 & -5.42 \\
$\mathbf{7 a}=\mathbf{7 b}$ & 2.73 & 0.48 & 10.34 & 2.51 \\
$\mathbf{8 a}=\mathbf{8 b}$ & -7.10 & -3.74 & 3.02 & 3.22 \\
$\mathbf{9 a}=\mathbf{9 b}$ & -2.06 & 1.70 & -2.39 & -4.18 \\
\hline
\end{tabular}

${ }^{\mathrm{a}} \mathrm{RS}=\Delta \mathrm{H}_{\mathrm{f}}($ keto, thione, imino $)-\Delta \mathrm{H}_{\mathrm{f}}$ (enol, thiol, amino). ${ }^{\mathrm{b}}$ Minus sign indicates the greater stabilities of keto, thione, imino forms. 
Table 3. Aqueous phase calculated acidity constants, $\mathrm{pK}_{\mathrm{a}}$, for the studied molecules

\begin{tabular}{|c|c|c|c|c|c|c|c|c|c|}
\hline & $\mathrm{MNDO}^{\mathrm{a}}$ & $\mathrm{AM} 1^{\mathrm{b}}$ & $\mathrm{PM}^{\mathrm{c}}$ & $\mathrm{PM}^{\mathrm{d}}$ & & $\mathrm{MNDO}^{\mathrm{a}}$ & $\mathrm{AM}^{\mathrm{b}}$ & $\mathrm{PM}^{\mathrm{c}}$ & $\mathrm{PM}^{\mathrm{d}}$ \\
\hline Equilibrium & $\mathrm{pK}_{\mathrm{a}}$ & $\mathrm{pK}_{\mathrm{a}}$ & $\mathrm{pK}_{\mathrm{a}}$ & $\mathrm{pK}_{\mathrm{a}}$ & Equilibrium & $\mathrm{pK}_{\mathrm{a}}$ & $\mathrm{pK}_{\mathrm{a}}$ & $\mathrm{pK}_{\mathrm{a}}$ & $\mathrm{pK}_{\mathrm{a}}$ \\
\hline $1 \mathrm{a}=1 \mathrm{Ha}$ & -6.17 & -2.29 & 2.99 & -13.19 & $\mathrm{~m} 1 \mathrm{a}=\mathrm{m} 1 \mathrm{Ha}$ & -6.02 & -3.46 & 1.09 & -12.89 \\
\hline $1 b=1 \mathrm{Hb}$ & -9.02 & 3.74 & 5.89 & -0.68 & $m 1 b=m 1 H b$ & -3.56 & 7.12 & 10.96 & -1.47 \\
\hline $2 \mathrm{a}=2 \mathrm{Ha}$ & -5.75 & -2.86 & 1.67 & -10.90 & $\mathbf{m} 2 \mathrm{a}=\mathrm{m} 2 \mathrm{Ha}$ & -8.59 & -3.42 & 1.30 & -12.83 \\
\hline $2 \mathrm{~b}=2 \mathrm{Hb}$ & 2.23 & 9.72 & 12.86 & 0.87 & $m 2 b=m 2 H b$ & -10.23 & 9.36 & 7.46 & -0.91 \\
\hline $3 \mathrm{a}=3 \mathrm{Ha}$ & -12.42 & -6.27 & -7.16 & -15.82 & $\mathrm{~m} 3 \mathrm{a}=\mathrm{m} 3 \mathrm{Ha}$ & -12.41 & -7.32 & -6.95 & -15.74 \\
\hline $3 b=3 H b$ & -2.06 & 8.18 & 11.39 & 0.40 & $m 3 b=m 3 H b$ & -3.54 & 11.67 & 10.92 & 0.47 \\
\hline $4 a=4 H a$ & -7.89 & -3.80 & 4.33 & -9.95 & $m 4 a=m 4 H a$ & -7.49 & -2.05 & 4.44 & -11.07 \\
\hline $4 b=4 H b$ & -6.55 & 7.20 & 8.38 & -0.93 & $m 4 b=m 4 H b$ & -6.21 & 8.55 & 7.25 & -0.12 \\
\hline $5 \mathrm{a}=5 \mathrm{Ha}$ & -7.73 & -0.36 & 4.74 & -9.62 & $\mathrm{m5a}=\mathrm{m} 5 \mathrm{Ha}$ & -10.03 & 0.01 & 4.94 & -10.45 \\
\hline $5 b=5 \mathrm{Hb}$ & -4.56 & 6.48 & 13.19 & -0.65 & $m 5 b=m 5 H b$ & -10.28 & 9.57 & 7.97 & -1.64 \\
\hline $6 a=6 \mathrm{Ha}$ & -11.49 & 3.31 & 6.32 & -9.67 & $\mathrm{~m} 6 \mathrm{a}=\mathrm{m} 6 \mathrm{Ha}$ & -11.69 & 3.22 & 4.90 & -11.92 \\
\hline $6 b=6 \mathrm{Hb}$ & -3.88 & 12.13 & 14.84 & 1.91 & $m 6 b=m 6 H b$ & -4.14 & 12.42 & 10.85 & 1.17 \\
\hline $7 a=7 \mathrm{Ha}$ & 2.76 & 23.97 & 19.04 & 7.76 & $\mathbf{m} 7 \mathbf{a}=\mathbf{m} 7 \mathrm{Ha}$ & 3.07 & 23.01 & 18.84 & 6.03 \\
\hline $7 b=7 \mathrm{Hb}$ & 0.86 & 7.76 & 15.95 & 5.35 & $m 7 b=m 7 H b$ & 1.12 & 17.55 & 14.76 & 4.38 \\
\hline $7 b^{\prime}=7 \mathrm{Hb}^{\prime}$ & -6.93 & 8.45 & 18.20 & 2.37 & $\mathbf{m}^{7} \mathbf{b}=\mathbf{m} 7 \mathbf{H b}^{\prime}$ & -12.85 & 2.29 & 8.38 & 4.79 \\
\hline $8 a=8 H a$ & 2.77 & 23.00 & 21.00 & 8.88 & $\mathrm{m8a}=\mathrm{m} 8 \mathrm{Ha}$ & 2.69 & 20.97 & 19.21 & 8.52 \\
\hline $8 b=8 H b$ & -8.88 & 16.06 & 14.76 & 1.88 & $\mathrm{~m} 8 \mathrm{~b}=\mathrm{m} 8 \mathrm{Hb}$ & -3.22 & 12.56 & 10.99 & 1.25 \\
\hline $8 b^{\prime}=8 \mathrm{Hb}^{\prime}$ & -5.99 & 8.50 & 16.90 & 10.42 & $m 8 b=m 8 H b^{\prime}$ & -5.42 & 11.10 & 13.89 & 8.13 \\
\hline $9 \mathrm{a}=9 \mathrm{Ha}$ & -0.39 & 16.43 & 18.35 & 3.00 & $m 9 a=m 9 H a$ & -8.09 & 16.54 & 19.49 & 3.63 \\
\hline $9 b=9 H b$ & -2.81 & - & 11.23 & 0.76 & $m 9 b=m 9 H b$ & -3.06 & - & 11.09 & 1.03 \\
\hline $9 \mathrm{~b}=9 \mathrm{Hb}^{\prime}$ & -2.17 & 15.79 & 20.57 & 8.07 & $m 9 b=m 9 H b^{\prime}$ & -6.89 & 10.39 & 11.21 & 6.66 \\
\hline
\end{tabular}

${ }^{\mathrm{a}} \Delta \mathrm{H}_{\mathrm{f}}: \mathrm{H}_{2} \mathrm{O}=-69.15 \mathrm{kcal} \mathrm{mol}^{-1} \cdot \mathrm{H}_{3} \mathrm{O}^{+}=35.34 \mathrm{kcal} \mathrm{mol}^{-1} ; \mathrm{S}: \mathrm{H}_{2} \mathrm{O}=44.95 \mathrm{cal} \mathrm{mol}^{-1} \mathrm{~K}^{-1} \cdot \mathrm{H}_{3} \mathrm{O}^{+}=$ $45.91 \mathrm{cal} \mathrm{mol}^{-1} \mathrm{~K}^{-1} \cdot{ }^{\mathrm{b}} \Delta \mathrm{H}_{\mathrm{f}}: \mathrm{H}_{2} \mathrm{O}=-68.45 \mathrm{kcal} \mathrm{mol}^{-1} \cdot \mathrm{H}_{3} \mathrm{O}^{+}=43.13 \mathrm{kcal} \mathrm{mol}^{-1} ; \mathrm{S} \mathrm{H}_{2} \mathrm{O}=45.11 \mathrm{cal}$ $\mathrm{mol}^{-1} \mathrm{~K}^{-1} \cdot \mathrm{H}_{3} \mathrm{O}^{+}=46.14 \mathrm{cal} \mathrm{mol}^{-1} \mathrm{~K}^{-1} \cdot{ }^{\mathrm{c}} \Delta \mathrm{H}_{\mathrm{f}}: \mathrm{H}_{2} \mathrm{O}=-61.88 \mathrm{kcal} \mathrm{mol}^{-1} \cdot \mathrm{H}_{3} \mathrm{O}^{+}=60.15 \mathrm{kcal} \mathrm{mol}^{-1} ; \mathrm{S}$ : $\mathrm{H}_{2} \mathrm{O}=45.01 \mathrm{cal} \mathrm{mol}^{-1} \mathrm{~K}^{-1} \cdot \mathrm{H}_{3} \mathrm{O}^{+}=45.99 \mathrm{cal} \mathrm{mol}^{-1} \mathrm{~K}^{-1} \cdot{ }^{\mathrm{d}} \Delta \mathrm{H}_{\mathrm{f}}: \mathrm{H}_{2} \mathrm{O}=-59.47 \mathrm{kcal} \mathrm{mol}^{-1} \cdot \mathrm{H}_{3} \mathrm{O}^{+}=$ $46.54 \mathrm{kcal} \mathrm{mol}^{-1} ; \mathrm{S}: \mathrm{H}_{2} \mathrm{O}=44.99 \mathrm{cal} \mathrm{mol}^{-1} \mathrm{~K}^{-1} \cdot \mathrm{H}_{3} \mathrm{O}^{+}=45.85 \mathrm{cal} \mathrm{mol}^{-1} \mathrm{~K}^{-1}$. 
Table 4. Aqueous phase calculated tautomeric equilibrium constants, $\mathrm{K}_{\mathrm{T}}$, for the tautomers

\begin{tabular}{|c|c|c|c|c|c|c|c|c|}
\hline & \multicolumn{2}{|c|}{ MNDO } & \multicolumn{2}{|c|}{ AM1 } & \multicolumn{2}{|c|}{ PM3 } & \multicolumn{2}{|c|}{ PM5 } \\
\hline Equilibrium & $\mathrm{pK}_{\mathrm{T}}^{\mathrm{a}}$ & $\mathrm{pK}_{\mathrm{T}}^{\mathrm{b}}$ & $\mathrm{pK}_{\mathrm{T}}^{\mathrm{a}}$ & $\mathrm{pK}_{\mathrm{T}}^{\mathrm{b}}$ & $\mathrm{pK}_{\mathrm{T}}^{\mathrm{a}}$ & $\mathrm{pK}_{\mathrm{T}}^{\mathrm{b}}$ & $\mathrm{pK}_{\mathrm{T}}^{\mathrm{a}}$ & $\mathrm{pK}_{\mathrm{T}}^{\mathrm{b}}$ \\
\hline $1 a=1 b$ & 3.97 & 2.46 & 10.73 & 10.57 & 8.97 & 9.87 & 12.41 & 11.42 \\
\hline $2 a=2 b$ & 8.02 & -1.64 & 9.22 & 12.81 & 11.25 & 6.16 & 13.16 & 11.91 \\
\hline $3 a=3 b$ & 3.28 & 8.87 & 2.48 & 15.88 & 5.49 & 17.87 & 5.13 & 16.21 \\
\hline $\mathrm{m} 1 \mathrm{a}=\mathrm{m} 1 \mathrm{~b}$ & 8.07 & & 10.93 & & 15.73 & & 15.73 & \\
\hline $\mathrm{m} 2 \mathrm{a}=\mathrm{m} 2 \mathrm{~b}$ & 8.42 & & 9.96 & & 13.23 & & 13.52 & \\
\hline $\mathbf{m} 3 \mathbf{a}=\mathbf{m} 3 \mathbf{b}$ & 8.65 & & 5.21 & & 12.18 & & 7.66 & \\
\hline $4 a=4 b$ & 1.33 & 1.28 & 7.85 & 7.77 & 4.91 & 3.99 & 9.67 & 10.96 \\
\hline $5 a=5 b$ & 4.30 & -0.25 & 4.48 & 7.01 & 6.65 & 3.02 & 8.09 & 8.80 \\
\hline $6 a=6 b$ & -2.17 & 7.55 & 0.09 & 6.75 & -8.03 & 5.96 & -1.72 & 13.10 \\
\hline$m 4 a=m 4 b$ & -6.65 & & -0.76 & & -0.94 & & 4.11 & \\
\hline $\mathrm{m} 5 \mathrm{a}=\mathrm{m} 5 \mathrm{~b}$ & -8.26 & & -1.09 & & 0.30 & & 2.93 & \\
\hline$m 6 a=m 6 b$ & -12.03 & & -6.63 & & -10.44 & & -5.71 & \\
\hline $7 a=7 b$ & -2.42 & $\begin{array}{l}-1.95 \\
-15.91\end{array}$ & -3.77 & $\begin{array}{c}-4.00 \\
-15.20\end{array}$ & -3.12 & $\begin{array}{c}-4.08 \\
-10.45\end{array}$ & -2.60 & $\begin{array}{l}-1.65 \\
-1.24\end{array}$ \\
\hline $8 a=8 b$ & -2.74 & $\begin{array}{l}-5.90 \\
-8.11\end{array}$ & -4.34 & $\begin{array}{l}-6.17 \\
-7.24\end{array}$ & -6.77 & $\begin{array}{l}-8.22 \\
-5.32\end{array}$ & 1.01 & $\begin{array}{l}-7.27 \\
-0.39\end{array}$ \\
\hline $9 a=9 b$ & -0.01 & $\begin{array}{l}5.02 \\
1.19\end{array}$ & -1.70 & $\begin{array}{c}- \\
-4.51\end{array}$ & -0.38 & $\begin{array}{l}-8.40 \\
-8.27\end{array}$ & -2.89 & $\begin{array}{r}-2.60 \\
3.03\end{array}$ \\
\hline $\mathbf{m} 7 \mathbf{a}=\mathbf{m} 7 \mathbf{b}$ & -3.72 & & -4.81 & & -2.91 & & -1.76 & \\
\hline $\mathrm{m} 8 \mathrm{a}=\mathbf{m 8 b}$ & 3.74 & & 1.41 & & 1.92 & & 1.68 & \\
\hline $\mathrm{m} 9 \mathrm{a}=\mathrm{m} 9 \mathrm{~b}$ & 8.83 & & -0.39 & & 0.04 & & 0.42 & \\
\hline
\end{tabular}

${ }^{\mathrm{a}} \mathrm{pK}_{\mathrm{T}}=\mathrm{pK}_{\mathrm{a}}($ product $)-\mathrm{pK}_{\mathrm{a}}($ reactant $)$ for parent compounds. ${ }^{\mathrm{b}} \mathrm{pK}_{\mathrm{T}}=\mathrm{pK}_{\mathrm{a}}($ model product $)-$ $\mathrm{pK}_{\mathrm{a}}$ (model reactant) for Charton equation [12].

Table 5. The aqueous phase computed HOMO and LUMO energies $(\mathrm{eV})$ of studied molecules

\begin{tabular}{ccccccccccccccccccc}
\hline \multicolumn{1}{c}{ MNDO } & \multicolumn{1}{c}{ AM1 } & \multicolumn{3}{c}{ PM3 } & & & PM5 & \\
\hline $\begin{array}{c}\text { Compo } \\
\text { und }\end{array}$ & HOMO & LUMO & $\mathrm{n}^{\mathrm{a}}$ & HOMO & LUMO & $\mathrm{n}^{\mathrm{a}}$ & HOMO & LUMO & $\mathrm{n}^{\mathrm{a}}$ & HOMO & LUMO & $\mathrm{n}^{\mathrm{a}}$ \\
\hline $\mathbf{1 a}$ & -10.41 & 0.60 & -11.01 & -9.68 & -0.22 & -9.46 & -9.72 & -0.68 & -9.04 & -9.71 & -1.02 & -8.69 \\
$\mathbf{1 b}$ & -10.09 & 0.34 & -10.43 & -9.41 & -0.15 & -9.26 & -9.72 & -0.71 & -9.01 & -9.50 & -0.96 & -8.54 \\
$\mathbf{2 a}$ & -10.16 & 0.64 & -10.80 & -9.44 & 0.12 & -9.56 & -9.66 & -0.28 & -9.38 & -9.43 & -0.42 & -9.01
\end{tabular}




\begin{tabular}{|c|c|c|c|c|c|c|c|c|c|c|c|c|}
\hline $2 b$ & -10.18 & 0.30 & -10.48 & -9.54 & 0.26 & -9.80 & -9.84 & -0.19 & -9.65 & -9.61 & -0.27 & -9.34 \\
\hline $3 a$ & -10.55 & -0.01 & -10.54 & -10.09 & -0.09 & -10.00 & -10.16 & -0.53 & -9.63 & -10.00 & -0.83 & -9.17 \\
\hline $3 b$ & -10.25 & 0.30 & -10.55 & -9.78 & 0.15 & -9.93 & -10.00 & -0.33 & -9.67 & -9.80 & -0.44 & -9.36 \\
\hline m1a & -10.31 & 0.56 & -10.87 & -9.58 & -0.25 & -9.33 & -9.86 & -0.73 & -9.13 & -9.59 & -1.07 & -8.52 \\
\hline m1b & -10.09 & 0.33 & -10.42 & -9.41 & -0.20 & -9.21 & -9.72 & -0.74 & -8.98 & -9.51 & -1.09 & -8.42 \\
\hline $\mathrm{m} 2 \mathrm{a}$ & -10.14 & 0.60 & -10.74 & -9.42 & 0.11 & -9.53 & -9.72 & -0.27 & -9.45 & -9.41 & -0.45 & -8.96 \\
\hline $\mathbf{m} 2 b$ & -10.12 & 0.31 & -10.43 & -9.49 & 0.17 & -9.66 & -9.75 & -0.24 & -9.51 & -9.44 & -0.58 & -8.86 \\
\hline m3a & -10.23 & -0.02 & -10.21 & -9.95 & -0.09 & -9.86 & -9.97 & -0.60 & -9.37 & -9.77 & -0.81 & -8.96 \\
\hline m3b & -10.31 & 0.25 & -10.56 & -9.69 & 0.12 & -9.81 & -9.96 & -0.37 & -9.59 & -9.79 & -0.44 & -9.35 \\
\hline $4 a$ & -9.99 & -0.67 & -9.32 & -9.67 & -0.90 & -8.77 & -10.06 & -1.34 & -8.72 & -9.71 & -1.08 & -8.63 \\
\hline $4 b$ & -10.05 & -0.08 & -9.97 & -9.45 & -0.30 & -9.15 & -9.74 & -1.15 & -8.59 & -9.43 & -1.09 & -8.34 \\
\hline $5 a$ & -10.03 & -0.65 & -9.38 & -9.51 & -0.85 & -8.66 & -9.88 & -1.20 & -8.68 & -9.47 & -0.77 & -8.70 \\
\hline $5 b$ & -10.15 & 0.13 & -10.28 & -9.44 & -0.26 & -9.18 & -9.82 & -0.74 & -9.08 & -9.39 & -0.90 & -8.49 \\
\hline $6 a$ & -9.96 & -1.24 & -8.72 & -9.62 & -1.52 & -8.10 & -9.98 & -2.29 & -7.69 & -9.47 & -1.51 & -7.96 \\
\hline $6 \mathrm{~b}$ & -10.12 & 0.27 & -10.39 & -9.41 & -0.01 & -9.40 & -9.81 & -0.83 & -8.98 & -9.42 & -0.64 & -8.78 \\
\hline m4a & -9.93 & -0.72 & -9.21 & -9.60 & -0.94 & -8.66 & -10.05 & -1.51 & -8.54 & -9.58 & -1.11 & -8.47 \\
\hline$m 4 b$ & -10.03 & -0.05 & -9.98 & -9.30 & -0.32 & -8.98 & -9.72 & -1.16 & -8.56 & -9.34 & -1.00 & -8.34 \\
\hline m5a & -9.97 & -0.70 & -9.27 & -9.51 & -0.89 & -8.62 & -9.90 & -1.36 & -8.54 & -9.45 & -0.77 & -8.68 \\
\hline m5b & -10.05 & -0.07 & -9.98 & -9.44 & -0.28 & -9.16 & -9.82 & -0.76 & -9.06 & -9.44 & -0.59 & -8.85 \\
\hline m6a & -10.00 & -1.25 & -8.75 & -9.59 & -1.55 & -8.04 & -9.90 & -2.32 & -7.58 & -9.40 & -1.52 & -7.88 \\
\hline m6b & -10.09 & 0.26 & -10.35 & -9.22 & 0.01 & -9.23 & -9.72 & -0.84 & -8.88 & -9.24 & -0.56 & -8.68 \\
\hline $7 a$ & -10.24 & 0.51 & -10.75 & -9.63 & -0.01 & -9.62 & -9.83 & -0.51 & -9.32 & -9.66 & -0.53 & -9.13 \\
\hline $7 b$ & -10.03 & 0.29 & -10.32 & -9.39 & 0.02 & -9.41 & -9.48 & -0.51 & -8.97 & -9.36 & -0.52 & -8.84 \\
\hline $8 a$ & -10.15 & 0.57 & -10.72 & -9.40 & 0.28 & -9.68 & -9.71 & -0.16 & -9.55 & -9.38 & -0.19 & -9.19 \\
\hline $8 b$ & -10.06 & 0.30 & -10.36 & -9.39 & 0.33 & -9.72 & -9.45 & -0.11 & -9.34 & -9.48 & -0.14 & -9.34 \\
\hline 9a & -10.27 & 0.32 & -10.59 & -9.79 & 0.15 & -9.94 & -9.93 & -0.36 & -9.57 & -9.75 & -0.35 & -9.40 \\
\hline $9 b$ & -10.26 & 0.30 & -10.56 & -9.76 & 0.22 & -9.98 & -9.89 & -0.31 & -9.58 & -9.60 & -0.17 & -9.43 \\
\hline $\mathbf{m} 7 \mathbf{a}$ & -10.02 & 0.68 & -10.70 & -9.63 & -0.01 & -9.62 & -9.72 & -0.50 & -9.22 & -9.58 & -0.55 & -9.03 \\
\hline m7b & -9.96 & 0.30 & -10.26 & -9.31 & 0.03 & -9.34 & -9.44 & -0.55 & -8.89 & -9.29 & -0.49 & -8.80 \\
\hline m8a & -10.01 & 0.70 & -10.71 & -9.38 & 0.25 & -9.63 & -9.72 & -0.19 & -9.53 & -9.38 & -0.17 & -9.21 \\
\hline m8b & -10.16 & 0.27 & -10.43 & -9.45 & 0.32 & -9.77 & -9.80 & -0.20 & -9.60 & -9.41 & -0.11 & -9.30 \\
\hline m9a & -9.99 & 0.31 & -10.30 & -9.73 & 0.16 & -9.89 & -9.79 & -0.36 & -9.43 & -9.65 & -0.31 & -9.34 \\
\hline m9b & -10.17 & 0.28 & -10.45 & -9.51 & 0.24 & -9.75 & -9.85 & -0.25 & -9.60 & -9.48 & -0.20 & -9.28 \\
\hline
\end{tabular}

${ }^{\mathrm{a}} \mathrm{n}$ is nucleophilicity and $\mathrm{n}=\mathrm{E}(\mathrm{HOMO})-\mathrm{E}(\mathrm{LUMO})$. 


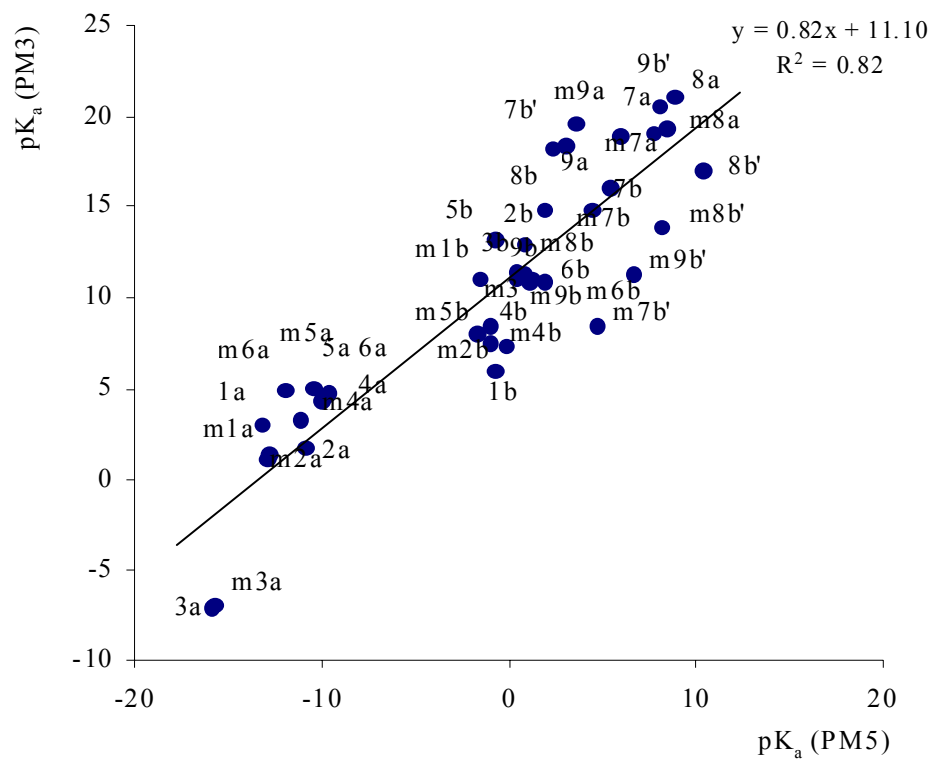

Figure 1. $\mathrm{pK}_{\mathrm{a}}$ correlation graphs.

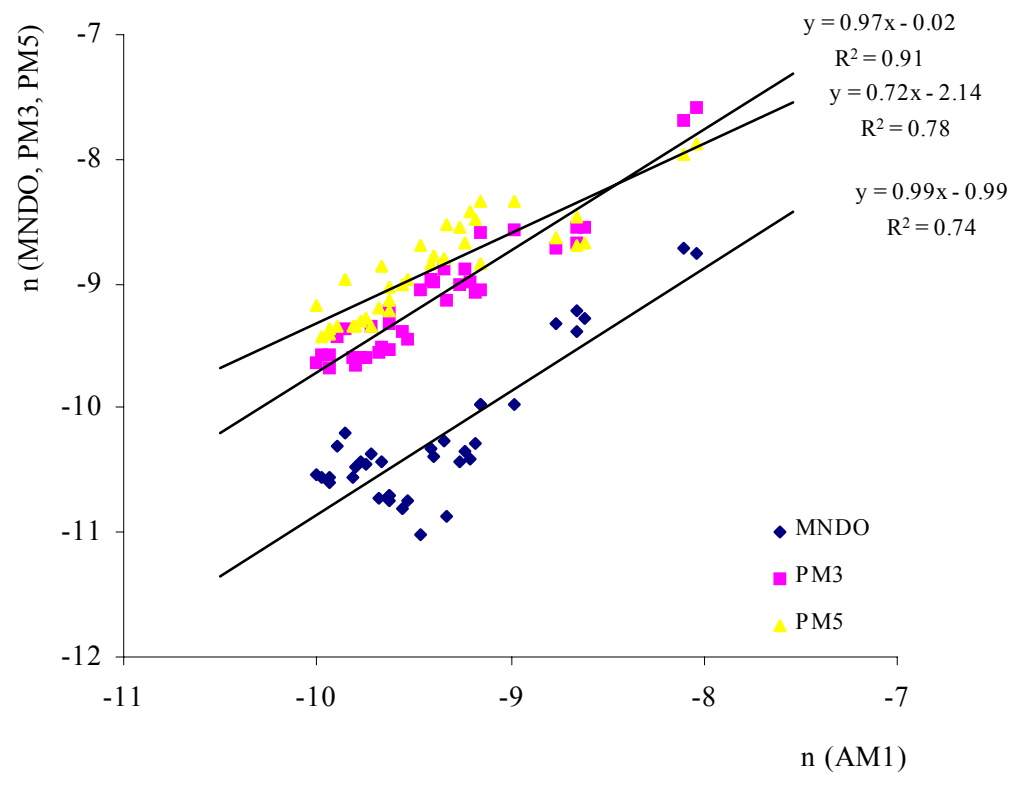

Figure 2. Nucleophilicity of the studied molecules. 


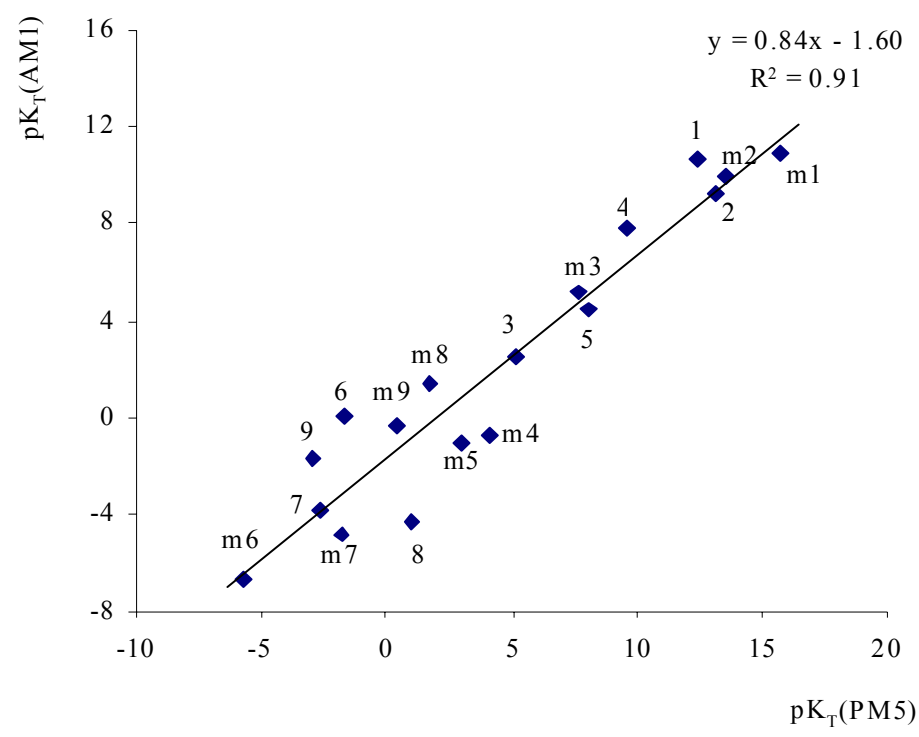

Figure 3. $\mathrm{pK}_{\mathrm{T}}$ correlation graph for studied molecules.

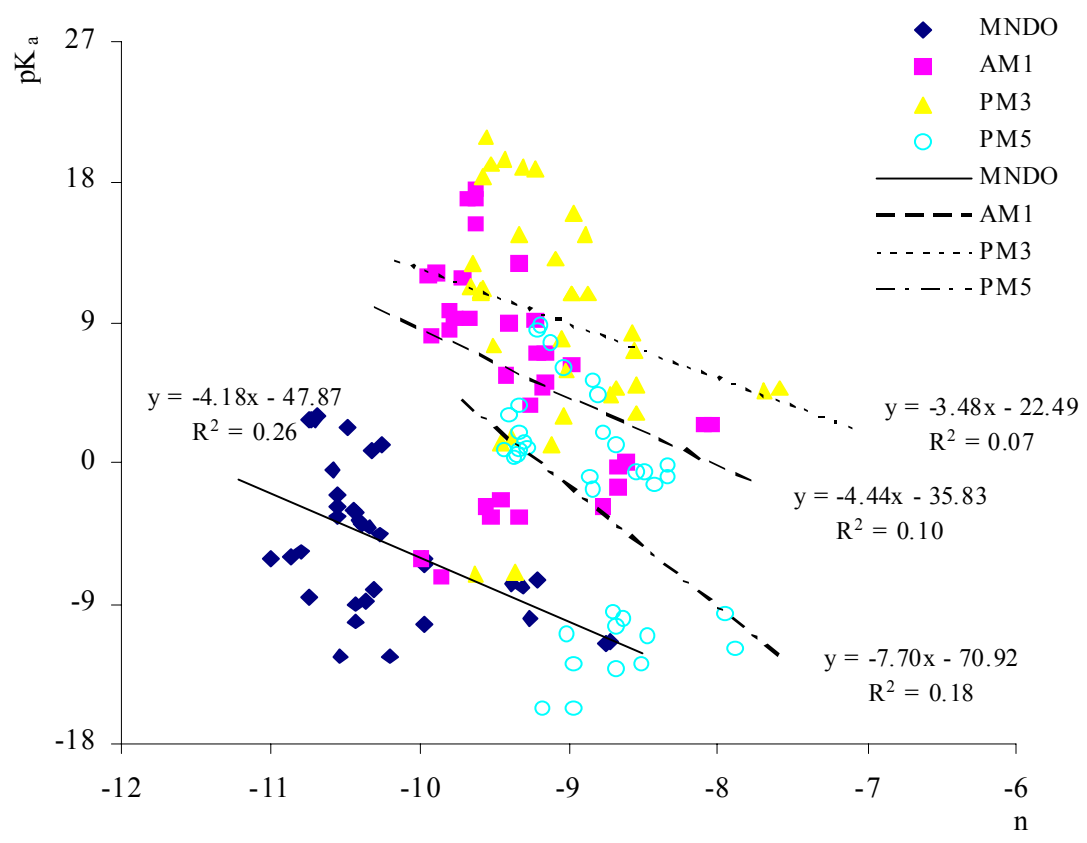

Figure 4. $\mathrm{pK}_{\mathrm{a}}-\mathrm{n}$ correlation graphs for studied molecules. 


\section{References}

1. Katritzky, A. R.; Pozharski, A.; Soldatenkov, A. T. Heterocycles in Life and Society, Wiley: NewYork, 1997.

2. Ogretir, C.; Tay, N. F.; Ozturk, I. I. accepted to be published in ARKIVOC.

3. Lim, C.; Bashford, D.; Karplus, M. J. Phys. Chem. 1991, 95, 5610.

4. Dewar, M. J. S.; Thiel, W. J. Am. Chem. Soc. 1977, 99, 4899

5. Dewar, M. J. S.; Zoebish, E. G.; Healy, E. F.; Stewart, J. J. P. J. Am.Chem. Soc. 1985, 107, 366.

6. Stewart, J. J. P. J. Comp. Chem. 1989, 109, 209.

7. Stewart, J. J. P. MOPAC 7.0 Quantum Chemistry Program Exchange, University of Indiana, Bloomington, In., USA.

8. Klamt, A.; Schüürman, G. J. Chem. Soc., Perkin Trans. 1993, 2, 799.

9. CS ChemOffice, Cambridge Scientific Computing Inc., Suite 61, 875 Massachusettes Avenue, Cambridge, MA 02139, USA.

10. CACHE SUPPLIER: Place and Date of Issue: Kraków, 27 September 2005FQS Poland Sp. z o.o. Starowiślna 13-1531-038 KrakowPoland

11. Speransa, M. Adv. Heterocy. Chem. 1985, 40, 25.

12. Katritzky, A. R. Physical Methods in Heterocyclic Chemistry, Academic Press:,1963, Vol.1, p 27.

13. Fravolini, A.; Grandolini, G.; Monzali, G. Ann. Chim. 1964, 54, 80.

14. Jensen, K. A.; Crossland, I. Acta Chem. Scand. 1963, 17, 144.

15. Filler, R.; Rao, Y.S. J. Org. Chem. 1962, 27, 3730.

16. Chanon, M.; Metzger, J. Bull. Soc. Chim. Fr. 1968, 7, 2855.

17. Kjellin, G.; Sandström, J. Acta Chem. Scand. 1969, 23, 2888.

18. Najer, H.; Armand, J.; Menin, J.; Voronine, N. C. R. Acta Sci. 1965, 260, 4343. 